\title{
Avian retrovirus integrase-enhanced transgene integration into mammalian cell DNA in vivo
}

\author{
Aqing Yao ${ }^{1}$, Roger Chiu ${ }^{2}$, Ajaykumar Vora ${ }^{2}$, David B. Brown ${ }^{1}$, \\ Duane Grandgenett ${ }^{2}$, and Brian R. Davis ${ }^{1}$
}

BioTechniques 35:1072-1078 (November 2003)

\begin{abstract}
Systems for introducing DNA genes-of-interest into mammalian cellular genomes have ranged from the use of different physical techniques to viruses including retroviruses. We have developed a microinjection method for an efficient and permanent integration of a DNA transgene into the cell genome by use of the retrovirus integrase. A 3.0-kb linear DNA fragment containing an internal herpes simplex virus thymdine kinase gene (tk) with flanking avian retrovirus U5 and U3 terminal attachment sites (U5-pgk/tk-U3) recognized by the integrase was constructed. The other donor, a 3.3-kb linear DNA fragment containing the same gene (pgk/tk) flanked by $\mathrm{ApaL} 1$ restriction sites not recognized by integrase, was also produced. After assembly of integrase-transgene complexes on ice, the complexes were microinjected into the nucleus of human fibroblast cells (143Btk-) containing a defective thymidine kinase. The number of hypoxanthine/aminopterin/thymidine (HAT)-resistant colonies produced upon microinjection of either naked DNA or the independently assembled integrase-transgene complexes were determined. Our data suggests that enhanced integration of U5-pgk/tk-U3 required the DNA attachment sites and co-delivery of integrase. The data was consistent with a direct role for both of these elements in producing an approximate 4-fold increase in the number of HAT-resistant colonies observed over microinjection of just naked U5-pgk/tk-U3 ( $\mathrm{P}<0.0001)$.
\end{abstract}

\section{INTRODUCTION}

Retroviruses have the capability of inserting their linear DNA genomes into host chromosomes. Upon reverse transcription of the viral RNA genome, the viral DNA along with viral proteins form large molecular weight nucleoprotein machines termed preintegration complexes (PIC) $(1,2)$. The two ends of the viral DNA are held together by a protein bridge presumably assisted by the viral integrase (IN) (2). IN removes two nucleotides from the blunt-ended viral genome, and subsequently, the PIC is transported into the nucleus (1). The 3' OH recessed viral DNA termini in the PIC are inserted by IN in a concerted fashion into the host genome producing proviral DNA. Insertion of the retrovirus genomes into the host chromosomes appears random although preferentially into active genes $(3,4)$.

Avian retroviruses integrate their DNA efficiently into mammalian chromosomes $(5,6)$. We investigated whether in vitro-assembled nucleo- protein complexes containing avian myeloblastosis virus (AMV) IN and a linear DNA fragment possessing a selectable gene-of-interest could be integrated directly into mammalian host chromosomes. The linear DNA donor contained avian retrovirus terminal attachment (att) site sequences that are recognized by IN for integration $(7,8)$. The delivery method for the assembled IN-DNA complexes was direct microinjection of the complexes into the cell nucleus. We produced a construct containing the herpes simplex virus (HSV) thymidine kinase $(t k)$ gene with terminal att sites (U5-pgk/tk-U3, $3.0 \mathrm{~kb}$ ) (Figure 1). For an additional naked DNA background control, the same pgk/tk construct lacking terminal att sequences was produced (pgk/tk, $3.3 \mathrm{~kb}$ ) (Figure 1). Human fibroblast cells $\left(143 \mathrm{Btk}^{-}\right)$containing a defective thymidine kinase were used as the host. We tested a variety of experimental protocols to examine whether both the att sites and IN were required to produce a significant increase in the number of hypoxanthine/aminopterin/ thymidine (HAT)-resistant colonies upon microinjection of assembled INDNA complexes. We present data demonstrating that co-delivery of IN with U5-pgk/tk-U3 results in a significant 3.9-fold increase in stable transduction $(P<0.0001)$ and that this significant increase is dependent on both att sites and IN.

\section{MATERIALS AND METHODS}

\section{Plasmid Constructs}

A plasmid construct containing the $t k$ gene driven by the phosphoglycerate kinase (pgk) promoter and flanked by att sequences was produced as follows. The expression vector $(\mathrm{pCMV} \beta$; BD Biosciences Clontech, Palo Alto, CA, USA) contained the lac $Z$ gene driven by the cytomegalovirus (CMV) promoter. The EcoRI, HindIII, SalI, and $P v u I I$ sites of pCMV $\beta$ were converted to PacI, SfiI, AscI, and FseI sites, re-

\footnotetext{
${ }^{1}$ University of Texas Medical Branch, Galveston, TX and ${ }^{2}$ Saint Louis University Health Sciences Center,
} Institute for Molecular Virology, St. Louis, MO, USA 
spectively, to produced pCMV9 $\beta$. The lac $Z$ gene in pCMV9 $\beta$ was replaced with the HSV $t k$ gene from pIC19/MCITK (provided by M.-J. Fackler, Johns Hopkins University School of Medicine, Baltimore, MD), and the CMV promoter sequences were replaced with the pgk promoter sequences (Ppgk) from pPGK-neo-bpA (provided by J. Ceci, University of Texas Medical Branch, Galveston, TX). ApaL1 digestion of this $\mathrm{pgk} / \mathrm{tk}$ construct produced a linear 3.3-kb fragment lacking att sites (Figure 1). Complementary 51-bp oligonucleotides corresponding to avian U5 (italicized)-NdeI site (bolded)-U3 (underlined) (5'-GCACCTGCATGAAGCAGAAGGCTTCATATGTAGTCTTATGCAATACTCTTG-3') and (5'-CAAGAGTATTGCATAAGACTACA TATGAAGCCTTCTGCTTCATGCAG$\left.G T G C-3^{\prime}\right)$ were synthesized to flank the Ppgk/tk sequences with att site sequences for IN recognition. The Ppgk/tk transgene was removed from the original $\mathrm{pgk} / \mathrm{tk}$ construct to produce a blunt-ended fragment that was ligated with the annealed oligonucleotides, digested with $N d e$ I, and subsequently cloned into the NdeI site of pUC19. The $\mathrm{pgk} / \mathrm{tk}$ transgene was released from pUC19 by NdeI digestion and was designated U5-pgk/tk-U3 (3.0 kb in length) (Figure 1). NdeI digestion produces linear DNA containing 3' $\mathrm{OH}$ recessed att sites necessary for strand transfer activity (8). DNA was isolated by $1 \%$ agarose gel electrophoresis and electro-eluted into dialysis tubing. The DNA was subsequently purified through a $0.1-\mu \mathrm{m}$ filter, extracted with phenol-chloroform, precipitated by ethanol, and dissolved into sterile TE (10 mM Tris- $\mathrm{HCl}, \mathrm{pH} 7.2,0.1 \mathrm{mM}$ EDTA). The DNA concentration was determined by absorbance at $280 \mathrm{~nm}$.

\section{Purified IN}

AMV IN was purified from virions to near homogeneity (9).

\section{Microinjection}

The 143Btk- human fibroblast cell line was obtained from ATCC (Manassas, VA, USA) and maintained in minimal essential medium containing $10 \%$ fetal bovine serum, penicillin/ streptomycin, and $15 \mu \mathrm{g} / \mathrm{mL}$ bromodeoxyuridine. Approximately $10^{4}$ 143Btk ${ }^{-}$cells were plated into $35-\mathrm{mm}$ dishes that were etched on the bottom surface to contain a grid pattern of 25 squares. Just prior to microinjection, cells were washed with media and fed with $2 \mathrm{~mL}$ of fresh media. Only one cell per square was injected with 50-100 cells injected per experiment.

The U5-pgk/tk-U3 DNA transgene was assembled with IN for approximately $15 \mathrm{~min}$ on ice in $3 \mathrm{mM}$ potassium phosphate-buffered solution $(\mathrm{pH}$ 7.2) containing $114 \mathrm{mM} \mathrm{KCl}$. No $\mathrm{Mg}^{2+}$ was present in the assembly buffer to help prevent integration of the DNA termini by IN into the same DNA molecule or adjacent DNA molecules prior to deliver into the nucleus. The IN dimer to DNA donor end molar ratio

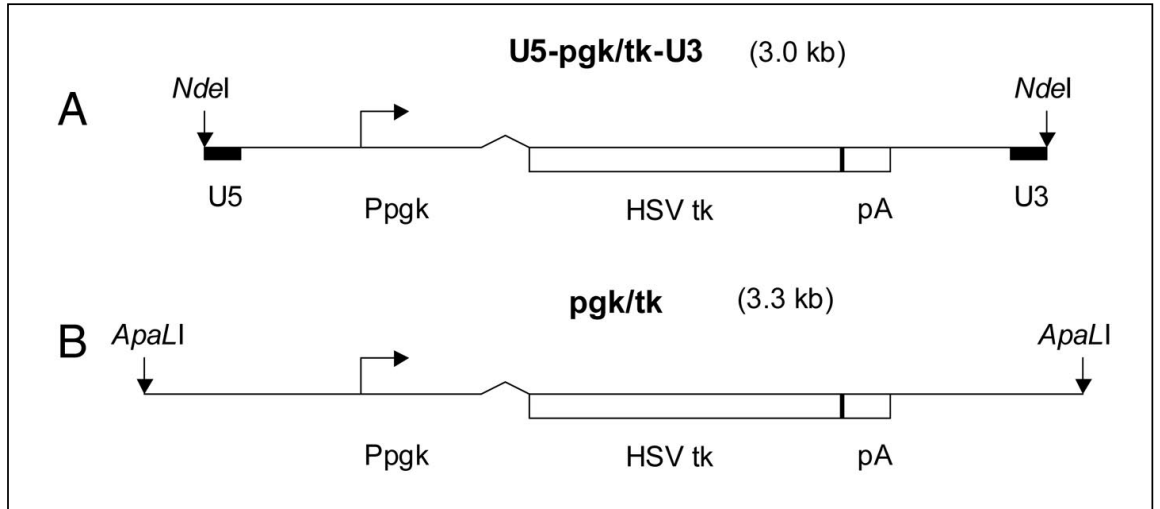

Figure 1. Diagram of the linear U5-pgk/tk-U3 and pgk/tk transgenes. (A) The HSV $t k$ gene driven by the pgk promoter is flanked by U5 and U3 att sites. Digestion of the pUC19 construct by NdeI site produced the appropriate $3^{\prime} \mathrm{OH}$ recessed termini for integration activity. The pA is the poly(A) tail. (B) Digestion of the original construct containing the pgk/tk gene by ApaLI produces the same transgene without the terminal att sites necessary for integration activity. was generally kept between 10:1 and 44:1, respectively, unless otherwise indicated. The assembled IN-U5-pgk/tkU3 complexes or corresponding naked DNA were microinjected with similar volumes (approximately $10 \mathrm{fL} /$ cell) directly into the nuclei of cells. At 24-72 $\mathrm{h}$ postinjection, the media was replaced with fresh HAT-containing medium, and the cells were incubated with new HAT medium every 3 days. At $10-15$ days postinjection, HAT-resistant colonies (approximately $5 \times 10^{2}$ to $10^{4}$ cells) in the individual squares were clearly identifiable and scored. There were no HAT-resistant colonies in the non-injected plates or in regions of the plate outside of the grid regions. For analysis of individual colonies or stable transductants, individual HATresistant colonies isolated by a cloning ring were trypsinized and expanded in HAT-containing medium for isolation of high molecular weight (HMW) genomic DNA.

\section{Statistical Analysis}

Data are presented as the mean of the sample populations \pm the standard deviation $(\bar{x} \pm \mathrm{SD})$. To test for significant difference between means, the Student's $t$-test (two tail) was employed. $P$ values were considered to be statistically significant if $<0.01$.

\section{Genomic DNA Analysis}

Individual isolated stable transductant cells were washed with phosphatebuffered saline (PBS) and suspended in extraction buffer $(10 \mathrm{mM}$ Tris- $\mathrm{HCl}$, $\mathrm{pH} 8.0,100 \mathrm{mM}$ EDTA) at $0.5 \times 10^{6}$ cell $/ \mathrm{mL}$. Sodium dodecyl sulfate (SDS) and RNaseA were added to a final concentration of $0.5 \%$ and $20 \mu \mathrm{g} / \mathrm{mL}$, respectively, for $1 \mathrm{~h}$ incubation at $37^{\circ} \mathrm{C}$. The samples were further digested with proteinase $\mathrm{K}$ (for $3 \mathrm{~h}$ at $56^{\circ} \mathrm{C}$ ), extracted several times with phenol-chloroform, precipitated by ethanol, and dissolved in TE buffer. Twenty micrograms of genomic DNA from each sample was digested with 200 U of HindIII. Digestion of genomic DNA with HindIII (not cutting within U5-pgk/tk-U3) yields fragments corresponding to individual integration events. The samples were subjected to electrophoresis in $1 \%$ aga- 
rose gels using a horizontal apparatus and transferred to Hybond ${ }^{\circledR} \mathrm{N}^{+}$membrane (Amersham Biosciences, Piscataway, NHJ, USA). After UV crosslinking, the membrane was hybridized with a ${ }^{32} \mathrm{P}$-labeled $t k$ gene probe. After processing, the membrane was exposed to X-ray film and developed.

\section{Inverse PCR Analysis}

The inverse PCR method by Nolta et al. (10) was applied to identify the host site 6-bp duplications observed upon concerted integration of the avian retrovirus genome in vivo (1). The reaction conditions, the sequence of the primers, and the DNA sequence of the approximately 230-bp PCR fragment containing the 6-bp host site duplication (AATTTG) identified in the host chromosome upon concerted insertion of U5-pgk/tk-U3 DNA by IN are available upon request.

\section{RESULTS}

\section{AMV IN Enhances Stable Transduc- tion of Human 143Btk- Fibroblasts with att Site-Flanked Transgene}

In order to investigate AMV IN-facilitated integration of DNA transgenes in vivo, we produced a linear $3.0-\mathrm{kb}$ DNA fragment containing an internal HSV $t k$ gene flanked by terminal U5 and $\mathrm{U} 3$ att sequences recognized by IN (Figure 1A). Stable transduction frequencies were determined for cells injected with U5-pgk/tk-U3 either in the absence (average $4.9 \% \pm 2 \%$ stable transduction frequency) or presence (average $18.5 \% \pm 3.9 \%$ ) of AMV IN (Table 1). Inclusion of AMV IN with the DNA yielded a significant 3.8-fold (average) increase in transduction $(P<$ $0.0001)$. The significance of this result is evident when the stable transduction frequency is normalized to the average survival frequency (47\%) of control 143Btk- cells injected with Oregon Green-coupled dextran (four experiments with a total of 250 cells injected) (11). Thus, taking this control survival factor into consideration, approximately $40 \%$ of cells surviving injection were stably transduced when AMV IN was co-delivered with U5-pgk/tk-U3.
We examined the role of the terminal att site sequences in IN-facilitated transgene delivery. The 3.0-kb U5-pgk/tk-U3 donor (Figure 1A) (Table 1) was compared to the similar 3.3-kb pgk/tk donor (Figure 1B) containing terminal ApaL1 sites. The U5-pgk/tk-U3 donor was delivered without (-IN) or with IN (+IN) (Table 2, left). The stable transduction frequency was $5 \% \pm$ $2 \%$ without IN in comparison to $20 \% \pm 5.8 \%$ with IN, a significant 4-fold (average) increase $(P=0.0007)$ similar to results shown in Table 1. For the pgk/tk donor lacking the terminal att sites, the stable transduction frequencies were $5.8 \% \pm 1.5 \%$ and $5.6 \%$ $\pm 3 \%$ without and with IN, respectively (Table 2 , right). Comparison of U5-pgk/tk-U3 delivered with IN to $\mathrm{pgk} / \mathrm{tk}$ was also significant $(P=0.0013)$ (Table 2). There was no significant difference in stable transduction frequency for naked U5-pgk/tk-U3 in comparison to naked $\mathrm{pgk} / \mathrm{tk}$ indicating that the att site sequences do not affect the stable transduction frequency. A significant difference in stable transduction frequency between the two DNA fragments was only apparent with IN present. The enhancement of stable transduction frequency requires both the att site sequences and IN, which is consistent with a direct role of both of these elements. The specific requirement for att sequences suggests that IN did not increase the stable transduction frequency for injected DNAs by simple coating of the DNA nonspecifically, thereby protecting it from nuclease degradation within the nuclei of injected cells.

We proceeded to evaluate the effect of DNA concentration on stable transduction frequency, both in the absence and presence of AMV IN. We utilized the U5-pgk/tk-U3 donor and an IN dimer to DNA end molar ratio of 20:1, respectively (see Figure 2). At the DNA concentrations examined $(1,4$, and 12 $\mathrm{ng} / \mu \mathrm{L})$, co-delivery of IN with U5-pgk/ tk-U3 DNA significantly increased the stable transduction frequency over that observed when just injecting U5-pgk/ tk-U3 $(P<0.0001)$ (Tables 1 and 2$)$. There was not a significant difference in transduction frequencies observed for the different naked DNA concentrations used for these experiments.

\section{Dependence of Enhanced Stable Transduction Frequency as a Function of IN Concentration}

To further investigate the enhanced transduction frequency of U5-pgk/tk$\mathrm{U} 3$ in the presence of IN, we examined the stable transduction frequency as a function of IN concentration, while maintaining a constant concentration of DNA ( $4 \mathrm{ng} / \mu \mathrm{L}$ or $2 \mathrm{nM})$. The stable transduction frequency was at a maximum for ratios of IN dimer per DNA at molar ratios greater than $8: 1$, respectively (Figure 2). Enhancement of concerted 
Table 2. Co-delivery of AMV IN With U5-pgk/tk-U3 but Not With pgk/tk Enhances Stable Transduction of 143Btk- Cells

\begin{tabular}{|c|c|c|c|c|c|c|}
\hline \multirow[b]{2}{*}{$\begin{array}{l}\text { Experiment } \\
\text { (No.) }\end{array}$} & \multicolumn{3}{|c|}{ U5-pgk/tk-U3 ${ }^{\mathrm{a}}$} & \multicolumn{3}{|c|}{ pgk/tk ${ }^{a}$} \\
\hline & $\begin{array}{l}-I N \\
(\%)\end{array}$ & $\begin{array}{l}+\mathrm{IN} \\
(\%)\end{array}$ & + IN/-IN & $\begin{array}{l}-I N \\
(\%)\end{array}$ & $\begin{array}{l}+\mathrm{IN} \\
(\%)\end{array}$ & $+\mathrm{IN} /-\mathrm{IN}$ \\
\hline 1 & 4 & 18 & $4.5 x$ & 6 & 10 & $1.7 \times$ \\
\hline 2 & 7 & 13.3 & $1.9 \times$ & 5 & 6 & $1.2 x$ \\
\hline 3 & 6 & 26 & $4.3 \times$ & 4 & 2 & $0.5 \times$ \\
\hline 4 & 6 & 16 & $2.7 \times$ & 8 & 4 & $0.5 \times$ \\
\hline 5 & 2 & 26 & $13 x$ & 6 & 6 & $1.0 \times$ \\
\hline Average $^{b}$ & $5.0 \pm 2.0^{c}$ & $20.0 \pm 5.8^{c}$ & $4.0 \times$ & $5.8 \pm 1.5^{c}$ & $5.6 \pm 3.0^{c}$ & $1.0 \times$ \\
\hline \multicolumn{7}{|c|}{$\begin{array}{l}\text { a Total number of HAT-resistant colonies as \% of total injected cell number. } \\
\text { b Average fold increase was calculated as the ratio of the average frequency } \\
+ \text { IN (20.0) to the average frequency -IN (5.0). }\end{array}$} \\
\hline \multicolumn{7}{|c|}{$\begin{array}{l}{ }^{c} \bar{x} \pm \mathrm{SD} \text {. The concentrations of DNAs in experiments } 1-5 \text { were } 1,1,6,6 \text {, and } \\
5 \mathrm{ng} / \mu \mathrm{L} \text {, respectively, and the DNAs were assembled with IN under normal } \\
\text { assay conditions. }\end{array}$} \\
\hline \multicolumn{7}{|c|}{$\begin{array}{l}\text { AMV, avian myeloblastosis virus; HAT, hypoxanthine/aminopterin/thymidine; } \\
\text { IN, viral integrase. }\end{array}$} \\
\hline
\end{tabular}

DNA integration events at similar AMV IN dimer to att DNA end molar ratios has been observed in vitro (12-14).

\section{Analysis of IN Mediated Integration of Transgene}

We examined individual HAT-resistant colonies for the number of integration events, the total number of gene copies integrated, and the organization

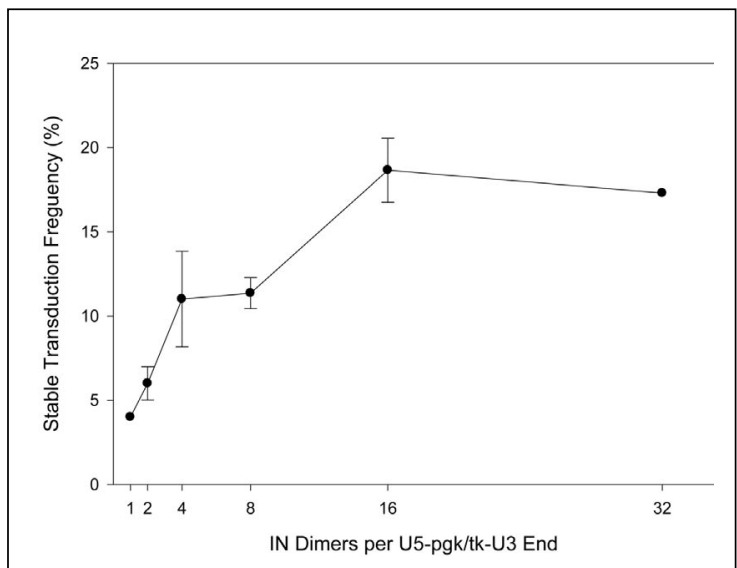

Figure 2. Enhanced transduction frequency is a function of the viral integrase (IN) concentration at a constant DNA concentration. IN was assembled with U5-pgk/tk-U3 at various IN dimers-to-donor end ratios. The DNA concentration was held constant for assembly at $4 \mathrm{ng} / \mu \mathrm{L}$ or $2 \mathrm{nM}$. The experiments were performed twice with the error bars indicating the repeated concentrations analyzed. Stable transduction frequency was determined as described in Table 1. of the integrated transgenes. HMW DNA isolated from individual expanded colonies was subjected to HindIII digestion, gel electrophoresis, Southern blot transfer, and hybridization with a ${ }^{32} \mathrm{P}$-labeled HSV $t k$ probe. All hybridizing bands should be $>3.0 \mathrm{~kb}$ (the size of U5-pgk/tk-U3), provided that there was no rearrangement of transgene sequences. A simple pattern of integration events is reflected in the HindIII digestion of DNA upon Southern blot analysis (Figure 3A) when the experiment was performed with U5-pgk/tk-U3 at $1 \mathrm{ng} / \mu \mathrm{L}$, corresponding to approximately three DNA copies delivered per injected cell (Table 1, experiment no. 4). HindIII analysis revealed an increase in the number of integration events per stable colony of cells in comparison to stable colony of cells without IN (Figure 3A). At $1 \mathrm{ng} / \mu \mathrm{L}$ DNA with IN, the number of transgene copies (approximately 1-3 copies of a specific internal transgene $1.6-\mathrm{kb} \quad B a m \mathrm{HI}$ fragment) observed in most stable colony sets (Figure 3B) were significantly 
lower than the number of transgene copies observed using $12 \mathrm{ng} / \mu \mathrm{L}$ DNA with IN (Figure 3D). HindIII analysis of the HMW DNA isolated from cells transduced at $12 \mathrm{ng} / \mu \mathrm{L}$ DNA with IN (Figure 3C) also revealed more complex patterns of integration sites than observed at $1 \mathrm{ng} / \mu \mathrm{L}$ DNA with IN (Figure $3 \mathrm{~A})$. In addition, we further analyzed each of the clones for the presence of head-to-tail concatamers by $\mathrm{NcoI}$ digestion. Since $N c o$ I cuts only once within the transgene, head-to-tail concatamers yield a diagnostic, $t k$-hybridizing 3.0$\mathrm{kb}$ fragment. At $12 \mathrm{ng} / \mu \mathrm{L}$ DNA with IN, significantly more integrated copies of U5-pgk/tk-U3 were also evident in all five colonies, either as single-copy genes or transgene concatamers, than at $1 \mathrm{ng} / \mu \mathrm{L}$ DNA with IN (data not shown). Thus, at the lower DNA concentration, IN-DNA complexes appear to form the desired structures mimicking the integration of viral DNA upon infection of cells by retroviruses.

Concerted integration of the avian retrovirus genome into the host genome results in a characteristic 6-bp duplication of host chromosomal DNA se- quences at the site of insertion (1). The duplication of host sequences is also observed upon concerted integration of similar DNA fragments containing terminal att site sequences by purified IN into target DNA in vitro (12-16). We evaluated the chromosomal integration sites for some of integration events utilizing inverse PCR (10). Although we were successful in individually cloning either upstream or downstream cellular flanking sequences adjacent to the inserted terminal att site for several individual integration events, we were only successful with one integration fragment in cloning both the upstream and downstream flanking sequences. The stable transductant for this one analysis was from the original HMW DNA used for HindIII analysis shown in Figure 3A, lane 5. Two individual HindIII integrated U5-pgk/tk-U3 bands (approximately 6.5 and $8.5 \mathrm{~kb}$ ) were identified. Nested inverse PCR yielded two products total: one upstream product of approximately $270 \mathrm{bp}$ (corresponding to the U5 sequence) and one downstream product of approximately $1000 \mathrm{bp}$ (corresponding to the $\mathrm{U} 3$ sequence). If the inverse PCR method had been completely successful, we would have expected a total of four DNA products: two upstream and two downstream. Sequencing of both the upstream and downstream product revealed a common "AATTTG" sequence flanking both $\mathrm{U} 5$ and $\mathrm{U} 3$. This suggested that both the upstream and downstream sequences flanked the same integrated DNA fragment and that the integration event occurred via an enzymatically active IN-U5-pgk/tkU3 complex. In order to confirm that the upstream and downstream flanking sequences from this genomic DNA sample corresponded to the same integration event, oligonucleotides corresponding to the upstream and downstream chromosomal sequences were synthesized. These primers were used to amplify normal chromosomal DNA of the expected size (approximately $230 \mathrm{bp}$ ) from the original $143 \mathrm{Btk}^{-}$cell line. Sequencing of this normal chromosomal DNA revealed the cellular sequence of 5'-TTATGTGTGAATTTGATCCTGTCGT-3' (the bolded nucleotides represent the adjacent cellular DNA sequences upstream and downstream

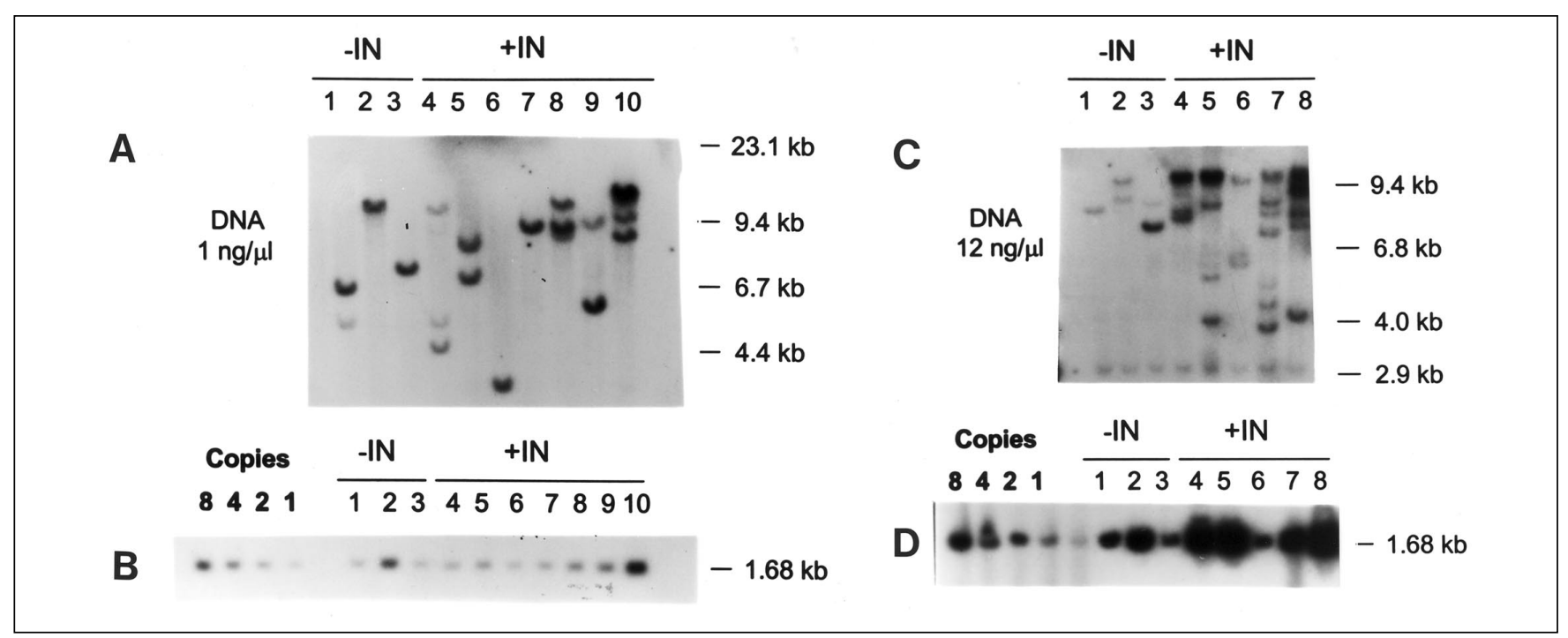

Figure 3. Viral integrase (IN)-facilitated integration of U5-pgk/tk-U3 at low and high DNA concentrations: analysis by HindIII digestion/Southern blot analysis and copy number determinations. (A) The DNA concentration for assembly with IN was $1 \mathrm{ng} / \mu \mathrm{L}$ (left). Each lane shows the HindIII/Southern blot analysis of high molecular weight (HMW) DNA isolated from individually expanded colonies. There are no HindIII sites within U5-pgk/tk-U3, and therefore, each hybridizing band reveals a separate integration event. Lanes 1-3 contain individual samples minus IN (-IN), and lanes 4-10 contain individual samples plus IN (+IN) present upon microinjection. The size markers indicated on the right were derived by HindIII digestion of $\lambda$ DNA. (B) For copy number determination, the same cellular DNA samples (lanes 1-10) were digested with BamHI, which releases a specific, internal 1.68-kb tk-hybridizing fragment from U5pgk/tk-U3. PhosphorImager TM (Amersham Biosciences) analysis was performed on the 1.68-kb band, and an X-ray film exposure is shown. There is approximately 1 transgene copy in lanes $1,3,4,6$, and 7 ; approximately 2 copies in lane 5 ; approximately 2.5 copies in lane 8 ; approximately $3-5$ copies in lanes 2 and 9; and $>8$ copies in lane 10. A set of the 1.68 -kb fragments of known copy numbers are shown on the left. (C) The DNA concentration for assembly with IN was $12 \mathrm{ng} / \mu \mathrm{L}$ (left). HindIII/Southern blot analysis of HMW DNA isolated from individually expanded colonies minus IN (-IN) (lanes 1-3) and plus IN (+IN)(lanes 4-8) are shown. (D) The same copy number analysis using the BamHI 1.68-kb band described above was performed on these samples. The fifth lane from the left had accidental spill over of the 1.68-kb standard. The copy number of transgenes in all the samples were significantly $>8$ copies except lanes 1,3 , and 6 . 
of the duplicated host site identified as AATTTG). This duplication of target chromosomal sequences demonstrated that the AMV IN delivered via microinjection was, in fact, enzymatically active and acted as expected for the insertion of viral DNA normally observed in virus-infected cells. In addition, the two other independently sequenced clones derived from a different colony set, each containing a different downstream viralhost junction, had the inserted terminal att CA dinucleotide joined to cellular sequences as well as the removal of the two unpaired nucleotides (AT) located at the $5^{\prime}$ ends of the att sites.

\section{DISCUSSION}

This is the first report that has successfully shown microinjected in vitro assembled IN-DNA transgene complexes being integrated into the host chromosomes. The microinjection data suggest that enhanced transgene integration was dependent upon both the att sites and IN, which is consistent with a direct role for both of these elements in producing the approximately 4-fold increase in the number of HAT-resistant colonies observed over microinjection of naked DNA only (Tables 1 and 2). Lipid vesicle-mediated DNA transfections of a linear U5-NeoR-U3 transgene $(1 \mu \mathrm{g})$ with a glutathione S-transferase (GST)-fusion avian retrovirus IN $(1 \mu \mathrm{g})$ into CHO-K1 cells produced a severalfold enhancement of stable transduction efficiency for G418 resistant cells (17). Our results differ from these prior results in several significant ways. Mizuarai et al. (17) observed enhanced transduction with IN when the NeoR construct was not flanked by att sites; suggesting, that their observed transduction effect may not be related to enzymatically active IN mediated integration. In contrast, the enhanced integration presently reported would only occur when flanking att sites were present arguing for specific interactions between IN and the att sites, which are necessary to mediate concerted DNA integration (Table 2). This specificity was confirmed by observing the 6-bp host site duplication mediated by specific host repair enzymes, which is a hallmark characteristic of enzymatically active retroviral integrase. Taken together, our results are consistent with the expected role of both att sites and IN in facilitating the increased integration rate. Further experiments are necessary to determine if there is a limitation with the length of the transgene using this approach. The integration of any transgene containing flanking att site sequences by co-delivery with IN into the male pronucleus of a fertilized egg may allow for more efficient production of animals containing low copy number of stable transgenes in their chromosomes $(18,19)$.

\section{ACKNOWLEDGMENTS}

The work was partially funded by a National Cancer Institute (NCI) grant (CA16312) to D.G. and a National Heart, Lung and Blood Institute (NHLBI) grant (R21DK053923) to B.D. We thank Peter Staniszlo for assistance with the molecular analysis, N.D. Victor Carsrud and M. Clara Carou who performed the microinjections, and J. Nolta for guidance with the inverse PCR protocol.

\section{REFERENCES}

1.Brown, P.O. 1997. Retrovirus integration, p. 161-203. In J. Coffin, S. Hughes, and H. Varmus (Ed.), Retroviruses. CSH Press, Cold Spring Harbor, NY.

2.Miller, M.D., C.M. Farnet, and F.D. Bushman. 1997. Human immunodeficiency virus type 1 preintegration complexes: studies of organization and composition. J. Virol. 71:5382-5390.

3.Schroder, A.R., P. Shinn, H. Chen, C. Berry, J.R. Ecker, and F. Bushman. 2002. HIV-1 integration in the human genome favors active genes and local hotspots. Cell 110:521-529.

4.Wu, X., Y. Li, B. Crise, and S.M. Burgess. 2003. Transcription start regions in the human genome are favored targets for MLV integration. Science 300:1749-1751.

5.Barsov, E.V. and S.H. Hughes. 1996. Gene transfer into mammalian cells by a Rous sarcoma virus-based retroviral vector with the host range of the amphotropic murine leukemia virus. J. Virol. 70:3922-3929.

6.Barsov, E.V., W.S. Payne, and S.H. Hughes. 2001. Adaptation of chimeric retroviruses in vitro and in vivo: isolation of avian retroviral vectors with extended host range. J. Virol. 75: 4973-4983.

7.Katz, R.A., G. Merkel, J. Kulkosky, J. Leis, and A.M. Skalka. 1990. The avian retroviral IN protein is both necessary and sufficient for integrative recombination in vitro. Cell 63:87-95.

8. Vora, A.C., M. McCord, M.L. Fitzgerald, R.B. Inman, and D.P. Grandgenett. 1994. Efficient concerted integration of retrovirus-like DNA in vitro by avian myeloblastosis virus integrase.
Nucleic Acids Res. 22:4454-4461.

9.Knaus, R.J., P.J. Hippenmeyer, T.K. Misra, D.P. Grandgenett, U.R. Muller, and W.M. Fitch. 1984. Avian retrovirus pp32 DNA binding protein. Preferential binding to the promoter region of long terminal repeat DNA. Biochemistry 23:350-359.

10.Nolta, J.A., M.A. Dao, S. Wells, E.M. Smogorzewska, and D.B. Kohn. 1996. Transduction of pluripotent human hematopoietic stem cells demonstrated by clonal analysis after engraftment in immune-deficient mice. Proc. Natl. Acad. Sci. USA 93:2414-2419.

11.Davis, B.R., J. Yannariello-Brown, N.L. Prokopishyn, Z. Luo, M.R. Smith, J. Wang, N.D.V. Carsrud, and D.B. Brown. 2000. Glass needle-mediated microinjection of macromolecules and transgenes into primary human blood stem/progenitor cells. Blood 96:437-444.

12.Vora, A.C. and D.P. Grandgenett. 1995. Assembly and catalytic properties of retrovirus integrase-DNA complexes capable of efficiently performing concerted integration. J. Virol. 69: 7483-7488.

13.Vora, A.C., R. Chiu, M. McCord, G. Goodarzi, S.J. Stahl, T.C. Mueser, C.C. Hyde, and D.P. Grandgenett. 1997. Avian retrovirus U3 and U5 DNA inverted repeats. Role Of nonsymmetrical nucleotides in promoting full-site integration by purified virion and bacterial recombinant integrases. J. Biol. Chem. 272:23938-23945.

14. Vora, A. and D.P. Grandgenett. 2001. DNase protection analysis of retrovirus integrase at the viral DNA ends for full-site integration in vitro. J. Virol. 75:3556-3567.

15.Aiyar, A., P. Hindmarsh, A.M. Skalka, and J. Leis. 1996. Concerted integration of linear retroviral DNA by the avian sarcoma virus integrase in vitro: dependence on both long terminal repeat termini. J. Virol. 70:3571-3580.

16. Hindmarsh, P., M. Johnson, R. Reeves, and J. Leis. 2001. Base-pair substitutions in avian sarcoma virus U5 and U3 long terminal repeat sequences alter the process of DNA integration in vitro. J. Virol. 75:1132-1141.

17.Mizuarai, S., M. Kamihira, K. Nishijima, and S. Iijima. 1999. Integrase-medicated nonviral gene transfection with enhanced integration efficiency. J. Biosci. Bioeng. 88:461-467.

18.Grandgenett, D.P., inventor. Saint Louis University, St. Louis, MO, assignee. In vitro method for concerted integration of donor DNA molecules using retroviral integrase proteins. US patent 5,888,270. Sept. 22, 1998.

19.Grandgenett, D.P., inventor. Saint Louis University, St. Louis, MO, assignee. Method for concerted integration of donor DNA molecules using retroviral integrase proteins. US patent US 6,316,261. Nov. 13, 2001.

Received 27 June 2003; accepted 3 September 2003.

Address correspondence to:

Duane Grandgenett

Saint Louis University Health Sciences Center

Institute for Molecular Virology

3681 Park Ave.

St. Louis, MO 63110, USA

e-mail: Grandgdp@slu.edu 